09

\title{
Ошибки измерений интерферометров с дифракционной волной сравнения
}

\author{
(c) А.А. Ахсахалян, Д.А. Гаврилин, И.В. Малышев, Н.Н. Салащенко, М.Н. Торопов, Б.А. Уласевич, \\ Н.Н. Цыбин, Н.И. Чхало
}

Институт фризики микроструктур РАН, 603950 Нижний Новгород, Россия

๑ e-mail: toropov@ipmras.ru

Поступило в Редакцию 28 марта 2019 г.

В окончательной редакции 28 марта 2019 г.

Принято к публикации 15 апреля 2019 г.

\begin{abstract}
Описаны экспериментальный стенд и результаты исследований аберраций источников эталонной сферической волны (ИЭСВ) на основе одномодового оптического волокна с субволновой выходной апертурой с использованием оптической части регистрирующей системы (ОЧРС). ИЭСВ и ОЧРС разрабатываются для безэталонного интерферометра с дифракционной волной сравнения. Описаны методики, позволяющие минимизировать ошибки измерений. Разработанные ИЭСВ и ОЧРС обеспечивают субнанометровую точность измерений оптики. Обсуждены возможности повышения точности измерений интерферометра до пикометрового уровня.
\end{abstract}

Ключевые слова: интерферометрия, аберрация, дифракционная волна сравнения, оптоволокно.

DOI: 10.21883/JTF.2019.11.48346.115-19

\section{Введение}

Оптические технологии являются ключевыми для развития практически всех высокотехнологичных отраслей. Тенденциями последних лет являются освоение коротковолнового диапазона длин волн и повышение разрешающей способности и функциональности оптоэлектронных систем. Укорочение длины волны, а также увеличение числа элементов в системе, кратно повысили требования к точности изготовления отдельных элементов и системы в целом. Необходимым условием создания оптических элементов с заданной точностью является адекватная метрология формы поверхностей и аберраций оптических систем. Основным методом измерений этих величин является интерферометрия. Чаще всего это интерферометры Физо. Суть метода заключается в том, что регистрируется интерферограмма от двух фронтов. Один фронт отражается от эталонной поверхности (эталонный фронт), второй - от исследуемой поверхности или прошедший через исследуемую систему (рабочий фронт). Аберрации рабочего фронта проявляются в виде искривления интерференционных полос. С помощью математической обработки интерферограмм с высокой точностью восстанавливаются величины аберраций и их тип [1]. Воспроизводимость результатов измерений может достигать $\lambda / 1000-\lambda / 3000$ ( $\lambda$ - длина волны интерферометра, соответственно воспроизводимость достигает долей нанометра) [2].

Ключевой проблемой традиционной интерферометрии является то, что в этом методе измеряются отклонения формы (аберрации) по отношению к эталону, иными словами, это относительные измерения. Качество эталонов практически ничем, кроме выданных сертифика- тов, не подтверждается. Это одна из причин, почему, зачастую, различные приборы при изучении одной и той же детали дают сильно различающиеся результаты, иногда на уровне $\lambda / 3-\lambda / 10$. Другая проблема использования эталонов заключается в том, что при их закреплении в механические оправы и последующей установке в приборы, поверхности неконтролируемым образом деформируются. Степень деформации сильно зависит от силы затяжки при установке. Как показывают эксперименты, эти деформации могут достигать уровня $\lambda / 5-\lambda / 50$. Имеются также ошибки эталонов, связанные с гравитацией. Например, эталон из кварца диметром $200 \mathrm{~mm}$, толщиной $50 \mathrm{~mm}$ под действием силы тяжести искажается на $20 \mathrm{~nm}$ (порядка $\lambda / 30$ ) [3]. Влияние гравитации на искажения формы поверхности зеркал посвящено много исследований, например, из последних работ $[4,5]$.

Проблема эталонов решается за счет использования интерферометров с дифракционной волной сравнения (ИДВС) [6-8]. В ИДВС сферическая волна сравнения (эталонная волна) формируется при дифракции света на малом, порядка длины волны, отверстии в непрозрачном экране, или на выходе одномодового оптического волокна. Качество дифракционной волны определяется хорошо изученными законами электродинамики. Более того, аберрации генерируемой волны могут быть измерены в опыте Юнга по интерференции двух волн от подобных источников [7]. Иными словами, этот тип интерферометра обладает функцией самокалибровки.

Однако эти интерферометры обладают рядом недостатков, сильно ограничивающих их широкое применение. В частности, ИДВС на основе одномодового оптического волокна [6] из-за большого диаметра кора $d$ 
(угловая апертура дифрагированной волны определяется отношением $\lambda / d$ ) обладают низкой рабочей апертурой и сильной неоднородностью интенсивности генерируемой волны. Помимо ограниченной апертуры сильная неоднородность интенсивности волны приводит к сдвигу координат экстремумов интерференционной картины и соответственно потере точности измерений [9].

Современные технологии литографии позволяют создавать отверстия нанометровых размеров. Это частично решает проблему повышения рабочей апертуры ИДВС и однородности интенсивности эталонной волны. Однако, как было показано в [8], аберрации генерируемой волны зависят от аберраций оптики, фокусирующей лазерное излучение на отверстие, и от ошибок при юстировке осей отверстия и лазерного пучка. Дополнительным фактором, снижающим качество дифрагированной волны, является шероховатость краев отверстия [10]. Физическим ограничением точности этого метода являются эффекты взаимодействия излучения с материалом экрана в краевой области. Из-за частичного прохождения излучения через край, возбуждения плазмон-поляритонных волн происходит аберрация дифрагированной волны [11].

Принципиально новый источник эталонной сферической волны (ИЭСВ), одномодовое оптическое волокно с субволновой выходной апертурой, был предложен авторами в [12]. На основе этого типа источника авторами и китайскими коллегами были созданы несколько образцов лабораторных интерферометров [11,13-16]. В работах показаны их достоинства. Однако декларируемая авторами точность измерений на уровне пикометров требует подтверждения, так как эти выводы сделаны на основании опыта Юнга по интерференции волн от двух ИЭСВ, с регистрацией интерференционной картины непосредственно на ПЗС (прибор с зарядовой связью) матрице $[11,14]$. Более того, авторы в [14] использовали матрицы с защитным стеклом, что, на наш взгляд, должно ограничивать точность измерений. Однако анализ возможных ошибок в составе ИДВС не был проведен. В частности на практике необходимо учитывать аберрации не только ИЭСВ, но и оптической части регистрирующей системы (ОЧРС), обеспечивающей функцию переноса изображения исследуемой детали на ПЗС матрицу. Так же ошибки могут возникнуть в результате некорректной юстировки положения источников, ОЧРС и центра ПЗС камеры (более строго центра контура, в котором ведется обработка интерференционной картины), наклона ПЗС матрицы относительно оптической оси регистрирующей системы ИДВС.

В настоящей работе описываются экспериментальный стенд и результаты исследований аберраций ИЭСВ на основе одномодового оптического волокна с субволновой выходной апертурой с помощью ОЧРС интерферометра, разрабатываемого авторами. Описываются методики, позволяющие минимизировать ошибки измерений.

\section{1. Описание стенда для измерений аберраций ИЭСВ}

Измерение аберраций ИЭСВ производилось на специально разработанном стенде, фотография которого приведена на рис. 1. Стенд располагается на оптическом столе с пневматической защитой от вибраций.

Работа стенда происходит следующим образом. Излучение $\mathrm{He}-\mathrm{Ne}$-лазера с помощью объектива заводится в одномодовое оптическое волокно. Далее в оптоволоконном делителе пучок делится на два в отношении по интенсивности 1:1. Каждый пучок проходит через поляризационный контроллер и затем в свой ИЭСВ. Для получения максимального контраста интерференционной картины на выходе ИЭСВ устанавливаются одинаковые поляризации света.

Далее сферические волны от каждого из источников, проходя через оптическую часть регистрирующей системы, интерферируют на ПЗС матрице. ОЧРС состоит из входного объектива и двух линз, согласующих размеры интерферирующих фронтов и ПЗС матрицы. Интерференционная картина передается в компьютер в цифровом виде, где производится обработка и восстановление аберраций волновых фронтов. В данном эксперименте использовался амплитудный режим регистрации интерферограмм, менее чувствительный к механическим вибрациям, шумам матрицы и интенсивности света [17].

Аберрации волновых фронтов $W(\mathbf{r})$ представляются в виде разложения по полиномам Цернике [18]:

$$
Z_{n}^{m}(r, \theta)=\sqrt{\frac{2(n+1)}{\pi\left(1+\delta_{m}^{0}\right)}} R_{n}^{m}(r) \Theta^{m}(\theta)
$$

— круговые полиномы Цернике, $\delta_{m}^{0}$ - дельта Кронекер,

$$
\begin{aligned}
\Theta^{m}(\theta) & = \begin{cases}\cos |m| \theta & (m>0) \\
1 & (m=0) \\
\sin |m| \theta & (m<0)\end{cases} \\
R_{n}^{m}(r) & =\sum_{s=0}^{(n-|m|) / 2} \frac{(-1)^{s}(n-s) !}{s !\left(\frac{n+m}{2}-s\right) !\left(\frac{n-m}{2}-s\right) !} r^{n-2 s}
\end{aligned}
$$

- радиальные полиномы,

$$
W(\mathbf{r})=\sum_{n=0}^{\infty} \sum_{\substack{m=-n \\ m+=2}}^{n} c_{n m} Z_{n}^{m}(r, \theta)
$$

— разложение функции $W(\mathbf{r})$,

$$
c_{n m}=\iint_{r \leq 1} W(\mathbf{r}) Z_{n}^{m}(r, \theta) d^{2} \mathbf{r}
$$

- коэффициенты разложения функции $W(\mathbf{r})$.

Использование этого формализма позволяет легко анализировать вклады в суммарный волновой фронт различных источников, деформирующих волновой фронт, 


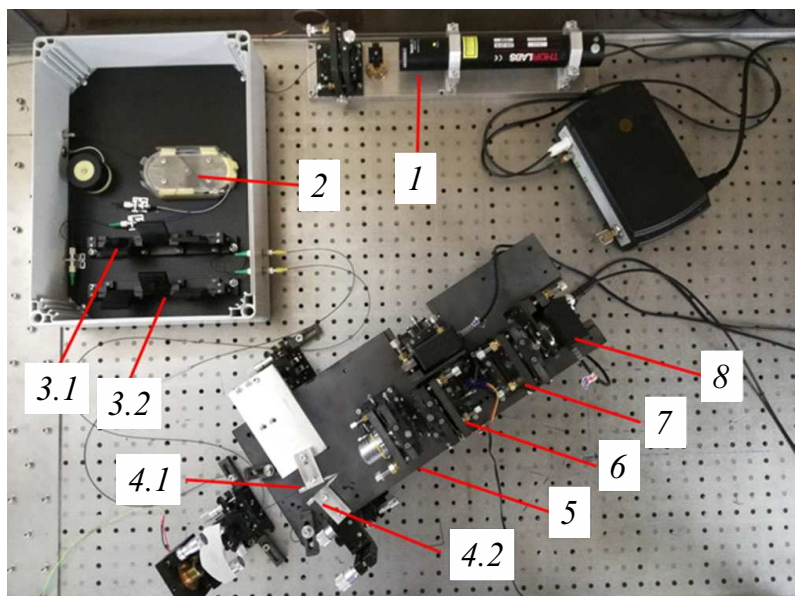

Рис. 1. Фотография стенда для изучения аберраций ИЭСВ. $1-\mathrm{He}-\mathrm{Ne}$-лазер с системой заводки излучения в одномодовое оптическое волокно; 2 - оптоволоконный делитель пучка на два канала; 3.1 и 3.2 - поляризационные контроллеры для каналов № 1 и № 2; 4.1 и 4.2 - ИЭСВ № 1 и № $2 ; 5$ объектив; 6 и 7 линзы № 1 и № 2, формирующие ОЧРС; 8 видеокамера на основе ПЗС матрицы.

например, аберрации ИЭСВ и ОЧРС, так как в суммарном разложении коэффициенты при соответствующих полиномах просто складываются:

$$
W(\mathbf{r})=W(\mathbf{r})_{\mathrm{SRSW}}+W(\mathbf{r})_{\mathrm{OPRS}} .
$$

В настоящей работе мы будем использовать 36-членный набор полиномов Цернике, который наиболее часто применяется различными авторами. Более подробно о связи степени полиномов Цернике и пространственными частотами, которые аппроксимирует данный набор полиномов, можно найти в [17].

Все требующие механической юстировки элементы стенда, такие как система заводки лазерного излучения в оптоволокно, оба ИЭСВ, объектив, обе линзы и видеокамера на основе ПЗС матрицы установлены на пяти координатных столах, обеспечивающих три линейных и два угловых перемещения. На столах предусмотрена жесткая фиксация положений после завершения юстировки.

\section{2. Описание ИЭСВ и ОЧРС}

Источник эталонной сферической волны представлял собой одномодовое оптическое волокно с субволновой выходной апертурой. Электронно-микроскопические изображения ИЭСВ приведены на рис. 2. Зауженный конец получался методом химического травления, металлизация боковых поверхностей производилась методом вакуумного напыления. Характерные выходные апертуры исследованных в настоящей работе образцов составляли $0.22-0.32 \mu \mathrm{m}$.
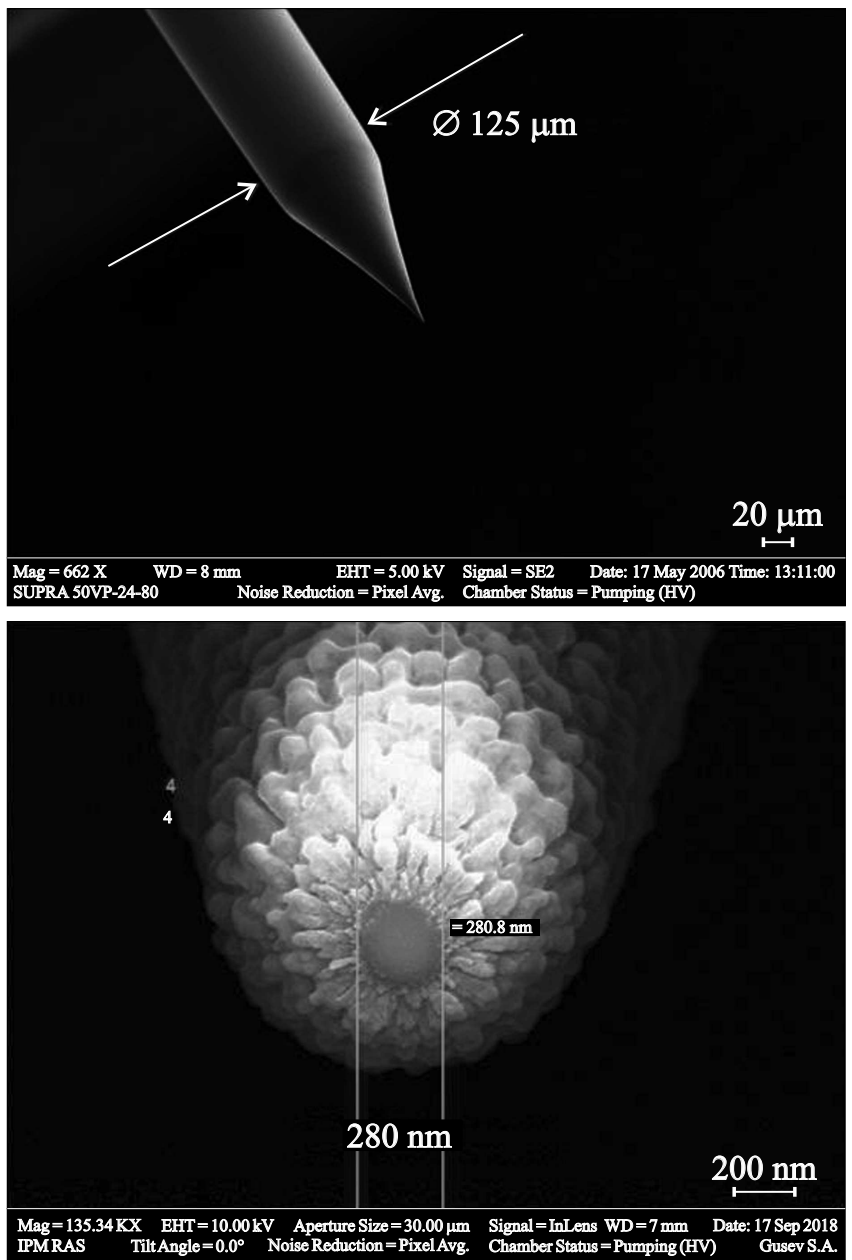

Рис. 2. Электронно-микроскопические изображения ИЭСВ на основе одномодового оптического волокна с субволновой выходной апертурой.

ОЧРС состоит из объектива Mitutoyo с рабочей числовой апертурой $N A=0.28$ и двух плоско-выпуклых линз, согласующих размеры выходной диафрагмы объектива и ПЗС матрицы (рис. 1). В таблице приведены основные геометрические параметры оптической схемы. Расчет оптической системы был проведен с использованием программы ZEMAX. Расчеты, проведенные для идеальных ИЭСВ, геометрических параметров линз и юстировки, показывают, что среднеквадратическая аберрация волнового фронта лежит в субнанометровой области.

\section{3. Методика измерений аберраций волновых фронтов ИЭСВ с ОЧРС}

Идею изучения аберраций волновых фронтов ИЭСВ иллюстрирует рис. 3. Для этих целей используется классическая схема Юнга по интерференции двух волн. Необходимо обратить внимание на то, что числовая апертура измеряемой волны ИЭСВ (оси источников направлены на противоположные краевые точки де- 
Параметры оптической схемы регистрирующей системы

\begin{tabular}{|c|c|c|c|c|c|}
\hline $\begin{array}{c}\text { Название } \\
\text { оптического } \\
\text { элемента или } \\
\text { поверхности }\end{array}$ & $\begin{array}{c}\text { Расстояние до } \\
\text { следующей } \\
\text { оптической } \\
\text { поверхности, } \\
\text { mm }\end{array}$ & $\begin{array}{c}\text { Радиус } \\
\text { кривизны, mm }\end{array}$ & $\begin{array}{c}\text { Полудиаметр, } \\
\text { mm }\end{array}$ & Среда & $\begin{array}{c}\text { Наклон к } \\
\text { оптической } \\
\text { оси, }{ }^{\circ}\end{array}$ \\
\hline $\begin{array}{c}\text { Волоконный } \\
\text { источник } \\
\text { сферической } \\
\text { волны }\end{array}$ & 276 & - & $2.5 \cdot 10^{-4}$ & Воздух & 20 \\
\hline $\begin{array}{c}\text { Входное окно } \\
\text { объектива } \\
\text { „Mitutoуо“ }\end{array}$ & 65 & - & 10 & - & 0 \\
\hline $\begin{array}{c}\text { Выходное окно } \\
\text { объектива } \\
\text { „Mitutoуо“ }\end{array}$ & 10 & - & 5.6 & - & 0 \\
\hline $\begin{array}{c}\text { Плоская грань } \\
\text { линзы } 1\end{array}$ & 5 & $\infty$ & 12.5 & К8 & 0 \\
\hline $\begin{array}{c}\text { Выпуклая } \\
\text { поверхность } \\
\text { линзы } 1\end{array}$ & 92.5 & -48.4 & 12.5 & К8 & 0 \\
\hline $\begin{array}{c}\text { Внутренний } \\
\text { фокус }\end{array}$ & 40 & - & - & - & 0 \\
\hline $\begin{array}{c}\text { Выпуклая } \\
\text { поверхность } \\
\text { линзы } 2\end{array}$ & 7 & 21 & 12.5 & К8 & 0 \\
\hline $\begin{array}{c}\text { Плоская грань } \\
\text { линзы } 2\end{array}$ & 53 & $\infty$ & 12.5 & К8 & 0 \\
\hline ПЗС камера & - & $\infty$ & 6 & ПЗС & 0 \\
\hline
\end{tabular}

тектора) примерно рана удвоенной числовой апертуре ОЧРС

$$
N A_{\mathrm{SRSW}} \approx 2 \times N A_{\mathrm{OPRS}}
$$

Расчет изображающих свойств и регистрирующей системы, а так же анализ влияния юстировки оптических элементов относительно друг друга на аберрации прошедших через ОЧРС фронтов ИЭСВ, был проведен с использованием программы ZEMAX. В программе моделируется интерференционная картина и проводится ее обработка в рамках формализма, приведенного в разд. 2.

На первой стадии с использованием этой программы были рассчитаны вклады аберрации комы, связанной с внеосевым расположением источников сферических волн, погрешности юстировки положения зондов, центра и наклона камеры по отношению к оптической оси в соответствующие члены разложения аберраций по полиномам Цернике. Тем самым, во-первых, определялись члены разложения, на которые наибольшее влияние оказывает анализируемый фактор; во-вторых, определялись минимальные аберрации каждого типа, которые определяются исключительно геометрией эксперимента, а не качеством волновых фронтов. Например, аберрации комы определяются расстояниями между источниками и между плоскостями источников и ПЗС матрицы. Смеще-

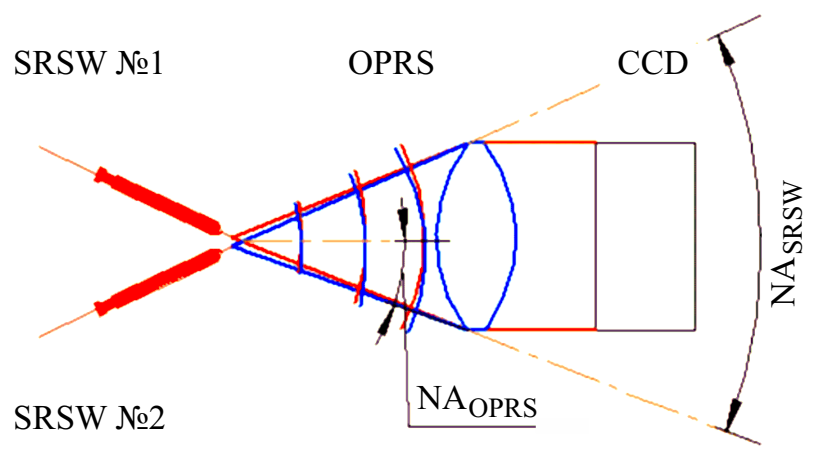

Рис. 3. Оптическая схема, поясняющая идею измерений аберраций ИЭСВ с использованием оптической части регистрирующей системы (опыт Юнга). ИЭСВ № 1 и ИЭСВ № 2 - источники эталонной сферической волны, ПЗС - ПЗС матрица, ОЧРС - оптическая часть регистрирующей системы. 
ние одного из ИЭСВ вдоль оптической оси приводит к появлению аберрации дефокусировка и астигматизм.

Грубая настройка (с точностью на уровне $100 \mu \mathrm{m}$ ) проводилась с помощью юстировочного лазера и регистрации изображений, в том числе теневых от ИЭСВ на ПЗС камере. Точная настройка осуществлялась с использованием интерферометрических измерений. На этом этапе настройка производилась таким образом, чтобы минимизировать соответствующие коэффициенты, рассчитанные на предыдущей стадии в разложении общей аберрации по полиномам Цернике. Таким образом, удается достичь наилучшей юстировки оптических элементов, тем самым обеспечив минимальные ошибки измерений.

\section{4. Экспериментальные результаты}

Разработанные стенд и методики были применены для изучения аберраций ИЭСВ с использованием ОЧРС. В эксперименте расстояние между ИЭСВ составляло $22 \mu \mathrm{m}$, числовая апертура ОЧРС $N A_{\mathrm{OPRS}}=0.28$, выходной зрачок объектива $11 \mathrm{~mm}$, размер чувствительной зоны детектора $6 \times 6 \mathrm{~mm}$. Так как оси источников направлены на крайние точки ОЧРС (рис. 3), то в эксперименте анализируются волновые фронты ИЭСВ, генерируемые в числовой апертуре $N A=\sin \left(32.52^{\circ}\right)$, или $N A \approx 0.54$.

На рис. 4 приведены интерферограмма с контуром, ограничивающим область обработки (рис. 4,a), из-

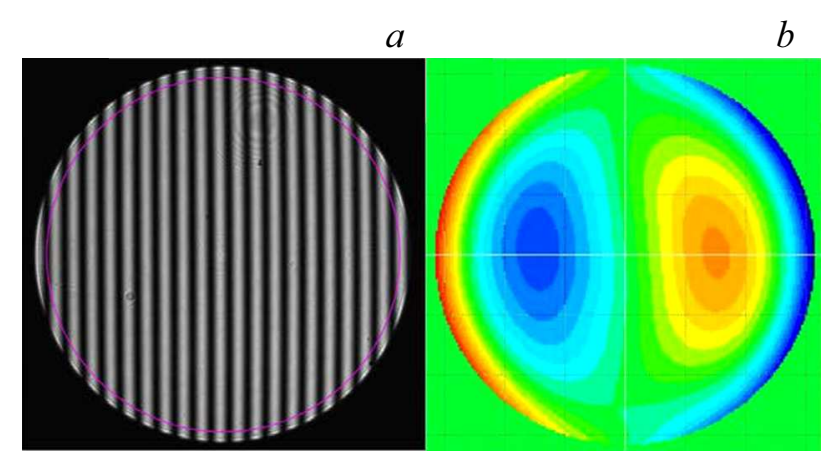

$c$

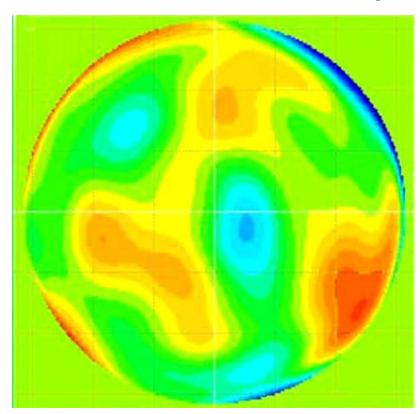

Рис. 4. Измеренная карта аберраций, контур ограничивает область обработки $(a)$, восстановленная по интерферограмме карта аберраций, $r m s=8.3 \mathrm{~nm}, P V=49 \mathrm{~nm}(b)$ и карта с убранной составляющей комы, связанной с внеосевым расположением ИЭСВ, $r m s=0.6 \mathrm{~nm}, P V=5.3 \mathrm{~nm}(c)$.

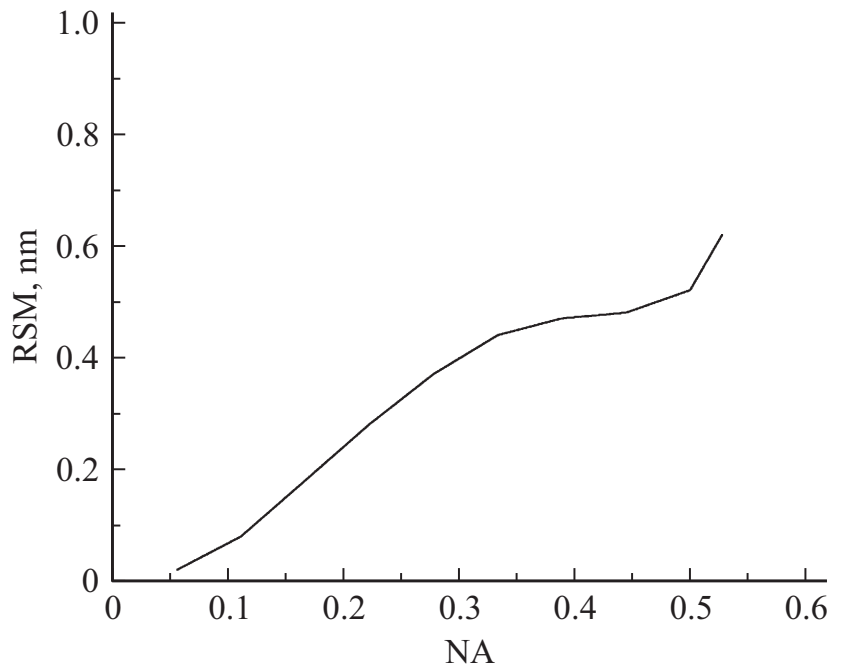

Рис. 5. Зависимость $r m s$ аберрации волнового фронта пары ИЭСВ, измеренные с использованием ОЧРС от числовой апертуры.

меренная карта аберраций, $r m s=8.3 \mathrm{~nm}, P V=49 \mathrm{~nm}$ (рис. 4,b) и карта с убранной составляющей комы, связанной с внеосевым расположением источников эталонной сферической волны, $r m s=0.6 \mathrm{~nm}, P V=5.3 \mathrm{~nm}$ (рис. $4, c)$.

Зависимость rms аберрации волнового фронта пары ИЭСВ, измеренные с использованием ОЧРС от числовой апертуры ИЭСВ, приведена на рис. 5. Эта зависимость определялась за счет пропорционального уменьшения области обработки интерферограммы. В действительности, это не совсем корректно, и если бы измерялись аберрации в области, симметричной по отношению к оси ИЭСВ, а не так как на рис. 3, где анализ производится вокруг угла относительно оси ИЭСВ около $16^{\circ}$, то они оказались бы существенно меньше. В настоящей работе они приводятся в таком виде, так как соответствующие части волнового фронта „работают“ в интерферометре.

Как видно из рис. 5 измеренные аберрации ИЭСВ растут по мере увеличения числовой апертуры. При этом $r m s$ аберраций находится на субангстремном уровне до $N A \approx 0.12$, и в целом не превышая $5 \AA$ до $N A \approx 0.5$.

\section{5. Обсуждение результатов и выводы}

В ходе проведенного исследования был получен ряд результатов, представляющих большое значение для практического применения интерферометрии с дифракционной волной сравнения, и для разработчиков соответствующей аппаратуры, в частности.

Во-первых, обнаружено сильное влияние взаимной юстировки оптических элементов ИДВС, даже для простейшей системы, состоящей из пары ИЭСВ и ПЗС видеокамеры, на результаты измерений. Так как эти точности находятся на микрометровом уровне, то для их 
удовлетворения требуется методическая работа, учитывающая специфику каждого прибора. В частности, в работе описывается многостадийная методика настройки с использованием на финальной стадии интерферометрии. Основой этой методики является теоретический анализ влияния каждой степени свободы для каждого оптического элемента на результирующий волновой фронт и определение вкладов в соответствующие коэффициенты разложения аберраций по полиномам Цернике, и их минимизация в процессе юстировки.

Во-вторых, несмотря на часто встречаемое мнение, что при дифракции света на малых апертурах генерируется „идеальная“ сферическая волна, на практике это не совсем так. Дефекты краев апертуры приводят к аберрациям волновых фронтов, которые усиливаются аберрациями различных оптических элементов как до дифракции, так и после нее. Таким образом, для достижения нанометровых, тем более ангстремных точностей измерений ИДВС необходима калибровка прибора. Лучшим методом калибровки является опыт Юнга по изучению интерференции от двух, близких по параметрам, ИЭСВ. Отбор таких источников производится так же в опыте Юнга.

В-третьих, в работе показано, что разработанные авторами ИЭСВ и ОЧРС напрямую обеспечивают субнанометровую точность измерений оптических элементов и систем с числовой апертурой до 0.28. Видно так же, что для обеспечения ангстремной и субангстремной точности требуется либо работать с меньшими угловыми апертурами, при этом прибегать к „сшивке“ большого числа кадров, либо использовать дополнительные методы, повышающие точность. Для повышения точности измерений до пикометрового уровня можно использовать несколько измерений с поворотом исследуемого объекта на фиксированные углы, например, как это было сделано в [13]. В этом случае решая систему уравнений можно исключить ошибки, вносимые аберрациями ИЭСВ и ОЧРС, которые не зависят от поворота детали.

\section{Благодарности}

Работа выполнена с использованием оборудования ЦКП ИФМ РАН. Финансирование работы

\section{Финансирование работы}

Финансирование работы при поддержке Минобрнауки РФ в рамках Соглашения № 075-02-2018-182 (RFMEFI60418X0202).

\section{Конфликт интересов}

Авторы заявляют, что у них нет конфликта интересов.

\section{Список литературы}

[1] Malacara D. Optical shop testing. 2nd ed. NY.: John Wiley \& Sons, Inc., 1992.

[2] Официальный вебсайт Zygo Corporation. URL: http://www.zygo.com

[3] Barysheva M.M., Chkhalo N.I., Pestov A.E., Salashchenko N.N., Toropov M.N., Zorina M.N. Fundamentals of Picoscience / Ed. Klaus D. Sattler, CRC Press, 2013. P. 595.

[4] Rhee H.-G., Kihm H., Yang H.-S., Ghim Y.-S., Lee Y.-W. // J. Korean Phys. Society. 2014. Vol. 65. N 9. P.1385-1389.

[5] Malyshev I.V., Chkhalo N.I., Toropov M.N., Salashchenko N.N., Pestov A.E., Kuzin S.V., Polkovnikov V.N. // Proc. SPIE. 2017. Vol. 10235. P. 102350C.

[6] Sommargren G.E. // Laser Focus World. 1996. Vol. 8. P. $61-71$.

[7] Naulleau P.P., Goldberg K.A., Lee S.H., Chang C., Attwood D., Bokor J. // Appl. Opt. 1999. Vol. 38. N 35. P. 7252-7263.

[8] Otaki K., Ota K., Nishiyama I., Yamamoto T., Fukuda Y., Okazaki S. // J. Vac. Sci. Technol. B. 2002. Vol. 20. N 6. P. 2449-2458.

[9] Chkhalo N.I., Pestov A.E., Salashchenko N.N., Toropov M.N. Lithography / Ed. Michael Wang, INTECH. (2010). URL: $\mathrm{http}$ ://sciyo.com/articles/show/title/manufacturing-and-investigating-objective-lens-for-ultrahigh-resolution-lithography-facilities

[10] Салащенко Н.Н., Торопов М.Н., Чхало Н.И. // Поверхность. Рентгеновские, синхротронные и нейтронные исследования. 2008. № 7. С. 3-5.

[11] Барышева М.М., Пестов А.Е., Салащенко Н.Н., Торопов М.Н., Чхало Н.И. // УФН. 2012. Т. 182. № 7. C. $727-747$.

[12] Chkhalo N.I., Klimov A.Yu., Rogov V.V., Salashchenko N.N., Toropov M.N. // Rev. Sci. Instrum. 2008. Vol. 79. P. 033107.

[13] Chkhalo N.I., Malyshev I.V., Pestov A.E., Polkovnikov V.N., Salashchenko N.N., Toropov M.N., Soloviev A.A. // Appl. Opt. 2016. Vol. 55. N 3. P. 619-625.

[14] Wang D., Chen X., Xu Y., Wang F., Kong M., Zhao J., Zhang B. // Opt. Express. 2014. Vol. 22. N 21. P. 25550-25559.

[15] Svechnikov M.V., Chkhalo N.I., Toropov M.N., Salashchenko N.N., Zorina M.V. // Opt. Lett. 2015. Vol. 40. N 2. P. 159-162.

[16] Wang D., Xu Y., Liang R., Kong M., Zhao J., Zhang B., Li W. // Opt. Express. 2016. Vol. 24. N 7. P. 7079-7090.

[17] Svechnikov M.V., Chkhalo N.I., Toropov M.N., Salashchenko N.N. // Opt. Express. 2015. Vol. 23. N 11. P. 14677-14694.

[18] Mahajan V.N. // Proc. SPIE. 2003. Vol. 5173. P. 1-17. 